\title{
Rhodiola Sacra Enhances Recovery from Sudden Deafness
}

Keywords: Hearing loss; Rhodiola; Oxidative stress

\begin{abstract}
Objectives: Ischemia and hypoxia are the important pathological mechanisms of Sudden Deafness (SD). Reactive Oxygen Species (ROS) are the important mediators of tissue injury during cochlear ischemia and reperfusion. Rhodiola Sacra (RS) is a typical traditional chinese medicine with property of anti hypoxia and antioxidant. This study was to observe the effect of RS on SD.

Patients and methods: Both of RS group and non-RS group contained 26 ears. Patients in RS group were treated with RS, and the patients in non-RS group were treated with placebo. Hearing gains of these two groups were compared. RS was taken orally at the dosage of $560 \mathrm{mg}$ each time, three times a day for 10 continuous days. There were no significant differences in clinical features and audiological data between RS and non-RS groups.
\end{abstract}

Results: The hearing gains of RS group at 250,500, 1000, 2000, and $4000 \mathrm{~Hz}$ were much higher than that of non-RS group correspondingly $(P<0.01)$. Also, the hearing gains of RS group at PTA (pure-tone average of $250,500,1000,2000$, and $4000 \mathrm{~Hz}$ ) were significantly higher than that of non-RS group $(P<0.01)$

Conclusion: The hearing gains of RS group were much higher than that of non RS group. RS is effective in the therapy of SD.

\section{Introduction}

Sudden Deafness (SD) is unknown cause sudden sensorineural hearing loss which involves three or more contiguous frequencies [1]. The most common causes of SD were vasculopathy, autoimmunity, viral infection and trauma. Acute disorder of inner ear blood supply is one of the most likely factors among these four possible pathogenic causes. Ischemia and hypoxia are the most important pathologic links in SD. Reactive Oxygen Species (ROS) take an important part in cochlear injury after ischemia and reperfusion; antioxidants definitely reduce cochlear damage induced by ischemia reperfusion [2-4]. There are no international guidelines accepted for the treatment of SD [5]. The therapeutic regimen of SD varies at different otology centers; current treatments include corticosteroid, vasodilator, antioxidant, anticoagulants, antiviral medications, traditional chinese medicine, acupuncture, and hyperbaric oxygen etc. Among these medications, corticosteroids are considered as the most effective therapy [6], and Hyperbaric Oxygen Therapy (HBOT) has been increasingly received attention $[7,8]$. HBOT can increase the partial oxygen pressure and improve microcirculation. HBOT makes much more oxygen dissolve in the blood through the increased partial oxygen pressure. It also improves the oxygen supply of cochlea, and this is the mechanism that HBOT is useful for the treatment of SD. With converse thinking, the medicine that can increase the hypoxia tolerance and utilization of oxygen should be useful for the treatment of SD.

Medicinal plant Rhodiola Sacra (RS) is very famous for its property of anti hypoxic effect. It can increase the hypoxia tolerance

\section{Otorhinolaryngology}

\author{
Chuanhong Yang ${ }^{1,2}$, Min Xiong ${ }^{2,3^{*}}$, Caihong $\mathrm{Li}^{2,3}$ and \\ Huangwen Lai, ${ }^{1,2}$
}

${ }^{\prime}$ Medical Research Department, Guangzhou General Hospital of Guangzhou Military Command, China

${ }^{2}$ Hearing Center, Guangzhou General Hospital of Guangzhou Military Command, China

${ }^{3}$ Department of Otolaryngology, Guangzhou General Hospital of Guangzhou Military Command, China

\section{*Address for Correspondence}

Min Xiong, Department of Otolaryngology, Guangzhou General Hospital of Guangzhou Military Command, Liu Hua Road 111, Guangzhou 510010, China; E-mail: dr_xiong@163.com

Submission: 11 September, 2017

Accepted: 13 October, 2017

Published: 16 October, 2017

Copyright: (๑) 2017 Yang C, et al. This is an open access article distributed under the Creative Commons Attribution License, which

and utilization of oxygen. It is a subtype of Rhodiola plants that can improve work efficiency, resist fatigue, relieve depression and prevent altitude sickness [9-13]. It has many pharmacological effects, and the most important pharmacological action is anti hypoxia and anti oxidation. Phytochemical investigations showed that RS contains about 19 antioxidants [14]. Among them, salidroside is the most important ingredient for the pharmacological action. Salidroside has significant antioxidant activity and its antioxidant capacity is concentration- and time-dependent [15]. Salidroside also has very strong anti hypoxia effect [16-20]. So, we speculated that RS has the treatment effect on SD. In this study we observed the treatment effect of RS on SD.

\section{Materials and Methods}

\section{Patients and evaluation}

The study consisted of RS group and non-RS group. All patients in this study received treatment in the Outpatient Department of Otolaryngology, Guangzhou General Hospital of Guangzhou Military Command. All the patients were in strict accordance with the diagnosis of SD. Informed consents were signed by all patients. And the time from hearing loss to treatment was less than 3 days. All patients received hearing tests including tympanometry, pure tone audiometry, and auditory brainstem evoked responses before treatment. In order to rule out brainstem lesions and acoustic tumors, magnetic resonance imaging of cerebellopontine angle and the internal auditory canal was done during treatment. Evaluation of hearing loss was assessed by the average hearing threshold at 250, 500,1000, 2000, and $4000 \mathrm{~Hz}$. The study was approved by the Ethics Committee of our hospital. In addition, exclusion criteria: (1) with history of noise exposure, ear disease, ototoxic drugs; (2) with systemic disease, such as hyperglycemia blood disease hypertension, and hyperlipemia etc.

\section{Patient groups and treatments}

The patients of this study were randomly divided into RS group and non-RS group. Each group contained 26 patients (26 ears). Patients 
were asked to quit smoking and drinking during the treatment. Also, patients were instructed to avoid mental work and have enough rest. The patients of both groups were intravenously treated with dexamethasone in the morning at the dosage of $0.2 \mathrm{mg} / \mathrm{kg} / \mathrm{d}$ for 10 successive days. Compound vitamin B was taken orally 2 tablets three times a day. $20 \mathrm{ml}$ danhong injection that is a typical vasodilator of traditional chinese medicine was also intravenously administrated $[21,22]$. The difference was that the RS group was treated with RS, and the non-RS group was treated with placebo. RS (Commodity name, Nuodikang Jiaonang, Tibet Nuodikang Pharmaceutical Limited Company, China; was approved by Chinese Drug Administration, approved Number:Z10980020) was administrated orally at the dosage of 560mg each time, three times a day for 10 continuous days.

\section{Hearing test}

The first hearing test was examined as soon as possible before any treatment. The pure-tone hearing thresholds were examined with GSI 61 clinical audiometer by a qualified hearing technician. Auditory brainstem evoked responses and tympanometry were also examined. The follow-up pure-tone hearing thresholds were taken when 10 days of treatment was finished. The patients who were not complete recovery in hearing during 10 days of treatment would receive an additional hearing test in 12 weeks. The hearing threshold measured at 12 weeks after starting treatment has been considered to be permanent threshold. Hearing losses were compared by using pure tone averaged thresholds of 250, 500, 1000, 2000, and $4000 \mathrm{~Hz}$. Absolute hearing gains of hearing thresholds were considered as the differences before and after treatment.

\section{Statistical analysis}

$\chi^{2}$ and $t$ tests were done by using SPSS 20.0 statistical software package.

\section{Results}

\section{Profile of patients}

All patients of both groups were with varying degrees of tinnitus and ear fullness, but with no vertigo. The profiles of the patients of both groups were showed in table 1 . There were no marked differences in age and gender between two groups. Also, there were no marked differences in hearing thresholds before treatment between RS and non-RS groups $(P>0.05)$.

Table 1: Profiles of patients.

\begin{tabular}{|c|c|c|c|}
\hline & RS group & non-RS group & $P$ \\
\hline No. of patients Age (years) & 26 & 26 & \\
\hline Mean \pm SEM & $42.25 \pm 8.66$ & $41.74 \pm 9.27$ & $0.759^{\mathrm{a}}$ \\
\hline Range & $33-52$ & $31-51$ & \\
\hline Gender (male/female) & $15 / 11$ & $12 / 14$ & $0.405^{\mathrm{b}}$ \\
\hline Vertigo & 0 & 0 & \\
\hline Tinnitus Initial hearing level(dB) & 26 & 26 & \\
\hline Mean \pm SEM(PTA) & $65.56 \pm 8.91$ & $63.21 \pm 6.42$ & $0.693^{\mathrm{a}}$ \\
\hline Range & $50-80$ & $50-80$ & \\
\hline Interval between onset and treatment \\
(days) & $\leq 3$ & $\leq 3$ & \\
\hline
\end{tabular}

PTA: Pure Tone Average of 250, 500, 1000, 2000 and $4000 \mathrm{~Hz}$. a t test; $b X^{2}$ test; RS: Rhodiola Sacra

\section{Therapeutic outcomes}

The therapeutic outcomes of two groups were showed in table 2. The average hearing gain of PTA was $34.58 \pm 8.51 \mathrm{~dB}$ and 22.05 $\pm 7.57 \mathrm{~dB}$ in RS and non-RS groups, respectively. It showed that the hearing gain in RS group was much higher than that of non-RS group $(P<0.01)$. Also, the hearing gain at $250,500,1000,2000$, and $4000 \mathrm{~Hz}$ in RS group was much higher than that of non-RS group correspondingly $(P<0.01)$.

\section{Discussion}

$\mathrm{SD}$ is not an independent disease; it is a symptom of cochlear disease. There is no specific treatment for SD currently, and comprehensive treatment has been advocated to improve the outcome. This study observed the effect of RS on SD. The hearing gains of 250, 500, 1000, 2000, $4000 \mathrm{~Hz}$ and PTA in RS group were marked higher than those of non-RS group $(P<0.01)$. The results have confirmed that RS has curative effect on SD.

Vascular dysfunction is considered to be the first pathogenic factor of SD. Blood disease or systemic vascular disease greatly increases the incidence of SD [23]. Decreasing blood pressure rapidly can induce SD in hypertensive patients [24]. Also, SD may be the precursor of cerebrovascular diseases $[25,26]$. Reducing the inner ear blood supply artificially can successfully make animal model of SD $[27,28]$. These indicate that disorders of cochlear blood supply are the main cause of SD. Cochlear hypoxia following ischemia occurs simultaneously, and it reaches a consensus that ischemia and hypoxia are the most important pathologic links in SD [27-32]. Ischemia-reperfusion definitely aggravates cochlear injury through the oxidative damage pathway $[2,33]$.

Hypoxia following ischemia reduces mitochondrial phosphorylation and formation of ATP, and produces much more superoxide. Blood oxygen content returns to normal level during reperfusion following ischemia. Much more superoxide has been produced by abundant oxygen during reperfusion. NADPH oxidase and xanthine oxidase can catalyze $\mathrm{O}_{2}$ with hydronium ions to create superoxide. ROS can directly damage DNA, lipids, proteins, and also damage cell through activating the caspase pathway. Also, nitric oxide synthase (NOS) takes important part in the cochlear damage following transient ischemia [33]. Inducible Nitric Oxide Synthase (iNOS) can catalyze the generation of large amounts of Nitric Oxide (NO) in damaged cochlea. Peroxynitrite $\left(\mathrm{ONOO}^{-}\right)$is produced when $\mathrm{NO}$ reacts with $\mathrm{O}^{-}$. ROS can also increase the formation of NO. ROS/NO can activate JNK pathway in the damaged cochlea [34]. JNK activates c-Jun that can induce the apoptosis of oxidative damaged cochlear cells [35].

Table 2: The hearing gains in $R S$ and non-RS group ( $n=26, d B H L)$.

\begin{tabular}{|c|c|c|c|}
\hline & RS group & non-RS group & $\boldsymbol{P}$ \\
\hline $250 \mathrm{~Hz}$ & $42.63 \pm 11.17$ & $30.85 \pm 10.48$ & $0.00412^{\mathrm{a}}$ \\
\hline $500 \mathrm{~Hz}$ & $33.52 \pm 9.52$ & $21.86 \pm 8.63$ & $0.00654^{\mathrm{a}}$ \\
\hline $1000 \mathrm{~Hz}$ & $32.92 \pm 7.68$ & $20.46 \pm 8.71$ & $0.00748^{\mathrm{a}}$ \\
\hline $2000 \mathrm{~Hz}$ & $31.48 \pm 6.52$ & $19.66 \pm 7.61$ & $0.00812^{\mathrm{a}}$ \\
\hline $4000 \mathrm{~Hz}$ & $30.32 \pm 8.39$ & $17.64 \pm 9.61$ & $0.00527^{\mathrm{a}}$ \\
\hline PTA & $34.58 \pm 8.51$ & $22.05 \pm 7.57$ & $0.00623^{\mathrm{a}}$ \\
\hline
\end{tabular}

PTA: Pure Tone Average of 250, 500, 1000, 2000 and $4000 \mathrm{~Hz}$. aRS group compared with non-RS group (t test). RS: Rhodiola Sacra 
RS is the typical representative of anti hypoxia drugs of traditional chinese medicine. RS has been used for preventing mountain sickness for thousands of years in china. Diving or high altitude can induce hypoxia in human body, trauma or other pathological damage factors also cause hypoxia in organs. RS has been widely used for treating the diseases related to hypoxia. For example, rhodiola has been widely used for angina pectoris and Ischemic Heart Disease (IHD) in China. Many clinical studies confirmed that symptoms of IHD could be relieved by rhodiola [36]. The exact mechanism of RS against hypoxia is not clear. Possible mechanisms are as follows: (1) salidroside increases glucose uptake and up regulates protein $\mathrm{O}-\mathrm{GlcNAc}$ and reduces cell damage induced by ischemia/reperfusion [17]; (2) salidroside inhibits Hypoxia-Inducible Factor (HIF)-1a expression and its translocation [19,37]; (3) salidroside inhibits mitochondria-dependent and fas-dependent apoptotic pathways [8]; (4) salidroside's property of oxidation resistance [38,39]. It is easy to understand that salidroside is effective for SD through its property of oxidation resistance [38-43]. The chemical formula of salidroside is $\mathrm{C}_{14} \mathrm{H}_{20} \mathrm{O}_{7}$, and molecular weight is 300.30 . Salidroside is a small molecular weight that can pass through the blood labyrinth barrier.

In addition, overwork and psychological stress seem to be the possible incentives for SD. RS has the function of anti fatigue, anti anxiety and anti depression [9-13]. Rhodiola sacra's improving the curative effect of SD may be also dependent on its property of anti fatigue, anti anxiety and anti depression.

\section{Conclusion}

We demonstrated that RS was positive to improve the recovery of SD. There are no published paper to explore the effects of RS on hearing loss currently. The most likely mechanisms of improving hearing from SD are through its property of anti hypoxia and anti oxidative damage, this can be demonstrated from previous publications about rhodiola plants research. The study that we have just finished confirmed that rhodiola sacra inhibited the expression of HIF-1 $\alpha$ and ROS in cochlea exposed to impulse noise and reduced cochlear injury. This demonstrated that rhodiola sacra reduced cochlear damage through its property of anti hypoxia and oxidation resistance.

\section{References}

1. Hughes GB, Freedman MA, Haberkamp TJ, Guay ME (1996) Sudden sensorineural hearing loss. Otolaryngol Clin North Am 29: 393-405.

2. Phlemiller KK, Dugan LL (1999) Elevation of reactive oxygen species following ischemia-reperfusion in mouse cochlea observed in vivo. Audio Neurootol 4: 219-228.

3. Tabuchi K, Nishimura B, Tanaka S, Hayashi K, Hirose Y, et al. (2010) Ischemia-reperfusion injury of the cochlea: pharmacological strategies for cochlear protection and implications of glutamate and reactive oxygen species. Curr Neuropharmacol 8: 128-134.

4. Seidman MD, Quirk WS, Nuttall AL, Schweitzer VG (1991) The protective effects of allopurinol and superoxide dismutase-polyethylene glycol on schemic and reperfusion-induced cochlear damage. Otolaryngol Head Neck Surg 1991 105: 457-463.

5. Rauch SD (2008) Clinical practice. Idiopathic sudden sensorineural hearing oss. N Engl J Med 359: 833-840.

6. Jarvis SJ, Giangrande V, John G, Thornton AR (2011) Management of acute diopathic sensorineural hearing loss: a survey of UK ENT consultants. Acta Otorhinolaryngol Ital 31: 85-89.
7. Sherlock S, Thistlethwaite K, Khatun M, Perry C, Tabah A (2016) Hyperbari oxygen therapy in the treatment of sudden sensorineural hearing loss: a etrospective analysis of outcomes. Diving Hyperb Med 46: 160-165.

8. Uzun G, Mutluoglu M, Metin S (2016) The use of hyperbaric oxygen treatment for sudden sensorineural hearing loss in Europe. Diving Hyperb Med 46: $43-$ 46.

9. Panossian A, Wikman G, Sarris J (2010) Rosenroot (Rhodiola rosea) traditional use, chemical composition, pharmacology and clinical efficacy. Phytomedicine 17: 481-493.

10. Cayer C, Ahmed F, Filion V, Saleem A, Cuerrier A, et al. (2013) Characterization of the anxiolytic activity of Nunavik Rhodiola rosea. Planta Med 79:1385-1391.

11. Amsterdam JD, Panossian AG (2016) Rhodiola rosea L. as a putative botanical antidepressant. Phytomedicine 23: 770-783,

12. Kang DZ, Hong HD, Kim KI, Choi SY (2015) Anti-Fatigue Effects of Fermented Rhodiola rosea Extract in Mice. Prev Nutr Food Sci 20: 38-42

13. Ma C, Hu L, Tao G, Lv W, Wang H (2015) An UPLC-MS-based metabolomics nvestigation on the anti-fatigue effect of salidroside in mice. J Pharm Biomed Anal 105:84-90

14. Ohsugi M, Fan W, Hase K, Xiong Q, Tezuka Y, et al. (1999) Active-oxygen scavenging activity of traditional nourishing-tonic herbal medicines and active constituents of Rhodiola sacra. J Ethnopharmacol. 67: 111-119.

15. Yu P, Hu C, Meehan EJ, Chen L (2007) X-ray crystal structure and antioxidant activity of salidroside, a phenylethanoid glycoside. Chem Biodivers 4: 508 513.

16. Lai MC, Lin JG, Pai PY, Lai MH, Lin YM, et al. (2014) Protective effect of salidroside on cardiac apoptosis in mice with chronic intermittent hypoxia. Int J Cardiol 174: 565-573.

17. Wu T, Zhou H, Jin Z, Bi S, Yang X, et al. (2009) Cardioprotection of salidroside from ischemia/reperfusion injury by increasing $\mathrm{N}$-acetylglucosamine linkage to cellular proteins. Eur J Pharmacol 613: 93-99.

18. Tan CB, Gao M, Xu WR, Yang XY, Zhu XM, et al. (2009) Protective effects of salidroside on endothelial cell apoptosis induced by cobalt chloride. Bio Pharm Bull 32: 1359-1363.

19. Li L, Qu Y, Jin X, Guo XQ, Wang Y, et al. (2016) Protective effect of salidroside against bone loss via hypoxia-inducible factor-1a pathwayinduced angiogenesis. Sci Rep 6: 32131.

20. Zheng KY, Zhang ZX, Guo AJ, Bi CW, Zhu KY, et al. (2012) Salidroside stimulates the accumulation of HIF-1a protein resulted in the induction of EPO expression: a signaling via blocking the degradation pathway in kidney and liver cells. Eur J Pharmacol 679: 34-39.

21. Wang H, Ren S, Liu C, Zhang X (2016) An Overview of Systematic Reviews of Danhong Injection for Ischemic Stroke. Evid Based Complement Alternat Med 2016: 8949835.

22. Yang M, Orgah J, Zhu J, Fan G, Han J, et al. (2016) Danhong injection attenuates cardiac injury induced by ischemic and reperfused neuronal cells through regulating arginine vasopressin expression and secretion. Brain Res 1642: 516-523.

23. Schweinfurth JM, Parnes SM, Very M (1996) Current concepts in the diagnosis and treatment of sudden sensorineural hearing loss. Eur Arch Otorhinolaryngol 253: 117-121.

24. Chao TK (2004) Sudden sensorineural hearing loss after rapid reduction of blood pressure in malignant hypertension. Ann Otol Rhinol Laryngol 113: 73 75.

25. Mun SK, Hong YH, Kang SH, Hwang SN (2010) A case of temporal ntracerebral hemorrhage that presented with sudden bilateral hearing loss as the initial symptom. J Korean Neurosurg Soc 48: 438-440.

26. Davidi ES, Warman M, Lahav Y, Halperin D (2010) Cerebellar ischemia nitially presented as sudden sensorineural hearing loss and gaze-evoked nystagmus. Isr Med Assoc J 12: 55-56. 
27. Asai Y, Umemura K, Kohno Y, Uematsu T, Nakashima M (1993) An anima model for hearing disturbance due to inner ear ischemia: photochemically nduced thrombotic occlusion of the rat anterior inferior cerebellar artery. Eur Arch Otorhinolaryngol 250: 292-296.

28. Schweinfurth JM, Cacace AT (2000) Cochlear ischemia induced by circulating ron particles under magnetic control: an animal model for sudden hearing oss. Am J Otol 21: 636-640.

29. Ondzotto G, Malanda F, Galiba J, Ehouo F, Kouassi B, et al. (2002) Sudden deafness in sickle cell anemia: a case report. Bull Soc Pathol Exot 95: 248 249.

30. Pujol R, Puel JL (1999) Excitotoxicity, synaptic repair, and functional recovery n the mammalian cochlea: a review of recent findings. Ann N Y Acad Sc 884: 249-254.

31. Sato K (1988) Pharmacokinetics of coenzyme Q10 in recovery of acute sensorineural hearing loss due to hypoxia. Acta Otolaryngol Suppl 458: 95 102.

32. Hansen S (1988) Postural hypotension--cochleo-vestibular hypoxiadeafness. Acta Otolaryngol Suppl 449: 165-169.

33. Tabuchi K, Tsuji S, Asaka Y, Hara A, Kusakari J (2001) Ischemia-reperfusion njury of the cochlea: effects of an iron chelator and nitric oxide synthase nhibitors. Hear Res 160: 31-36.

34. Nagashima R, Yamaguchi T, Tanaka H, Ogita K (2010) Mechanism underlying the protective effect of tempol and Nw-nitro-Larginine methyl ester on acoustic injury: possible involvement of c-Jun N-terminal kinase pathway and connexin26 in the cochlear spiral ligament. J Pharmacol Sci 114: 50-62.

35. Zine A, van de Water TR (2004) The MAPK/JNK signaling pathway offers potential therapeutic targets for the prevention of acquired deafness. Curr Drug Targets CNS Neurol Disord 3: 325-332.
36. Yu L, Qin Y, Wang Q, Zhang L, Liu Y, et al. (2014) The efficacy and safety of Chinese herbal medicine, Rhodiola formulation in treating ischemic heart disease: a systematic review and meta-analysis of randomized controlled trials. Complement Ther Med 22: 814-825.

37. Wu YL, Piao DM, Han XH, Nan JX (2008) Protective Effects of Salidroside against Acetaminophen-Induced Toxicity in Mice. Biol Pharm Bull 31: 15231529.

38. Zhong X, Lin R, Li Z, Mao J, Chen L (2014) Effects of Salidroside on cobalt chloride-induced hypoxia damage and mTOR signaling repression in PC12 cells. Biol Pharm Bull 37: 1199-1206.

39. Lee SY, Shi LS, Chu H, Li MH, Ho CW, Lai FY, Huang CY, Chang TC (2013) Rhodiola crenulata and Its Bioactive Components, Salidroside and Tyrosol, Reverse the Hypoxia-Induced Reduction of Plasma-Membrane-Associated Na,K-ATPase Expression via Inhibition of ROS-AMPK-PKC $\xi$ Pathway. Evid Based Complement Alternat Med 2013: 284150.

40. Capaccio P, Pignataro L, Gaini LM, Sigismund PE, Novembrino C, et al (2012) Unbalanced oxidative status in idiopathic sudden sensorineural hearing loss. Eur Arch Otorhinolaryngol 269: 449-453.

41. Hatano M, Uramoto N, Okabe Y, Furukawa M, Ito M (2008) Vitamin E and vitamin $\mathrm{C}$ in the treatment of idiopathic sudden sensorineural hearing loss. Acta Otolaryngol 128: 116-121.

42. Joachims HZ, Segal J, Golz A, Netzer A, Goldenberg D (2003) Antioxidants in treatment of idiopathic sudden hearing loss. Otol Neurotol 24: 572-575.

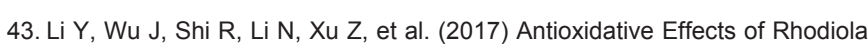
genus: Phytochemistry and Pharmacological Mechanisms against the Diseases. Curr Top Med Chem 17: 1692-1708.

\section{Acknowledgements}

The authors thank Professor Dingrong Zhou for her guidance. The study was supported by the National Natural Science Foundation of China (NO. 81470702).Wei Wang: Statistical review of data. 